\title{
Aggressive management of doxorubicin-induced cardiomyopathy associated with 'low' doses of doxorubicin
}

\author{
S. Sriskandan, M.E.R. O’Brien, I.E. Smith, P. Collins' ${ }^{1}$ and M.E. Gore \\ Department of Medicine, Royal Marsden Hospital, Fulham Road, London SW3 $6 J J$ and ' Department of \\ Cardiology, The Royal Brompton Heart and Lung Hospital, Fulham Road, London SW3 6NP, UK
}

\begin{abstract}
Summary: There is a dose-effect relationship between doxorubicin and the incidence of symptomatic cardiac failure. It is generally thought that doses below $500-550 \mathrm{mg} / \mathrm{m}^{2}$ are safe but, when objective measures of cardiac function are used, it becomes apparent that degrees of cardiac failure occur at doses below this. We present here the case histories of two patients who developed very severe cardiac failure at cumulative doses well below $500 \mathrm{mg} / \mathrm{m}^{2}$ but who survived their initial cardiac illness due to aggressive intervention. In one case, the patient was successfully treated by orthotopic cardiac transplantation.
\end{abstract}

\section{Introduction}

Clinically evident cardiotoxicity following treatment with the anthracycline doxorubicin is well documented. It is dose related and usually develops after a cumulative dose of $>500 \mathrm{mg} / \mathrm{m}^{2}$ has been administered. ${ }^{1}$ This side effect is becoming increasingly important as more patients with childhood cancers, leukaemias and lymphoma are cured by chemotherapy combinations that include this drug.

In this communication we question the notion of a 'safe' cumulative dose of doxorubicin by reporting two cases of severe dilated cardiomyopathy occurring in patients who had received cumulative doses that were within the 'non-cardiotoxic' range. One of these patients has received cardiac transplantation.

\section{Case 1}

A 51 year old Nigerian woman presented with a 2 year history of night sweats and massive lymphadenopathy in the left axilla and left supraclavicular fossa. In addition, there was a left pleural effusion and she was hypercalcaemic. She was a non-smoker and her blood pressure at presentation was $130 / 80 \mathrm{mmHg}$. Lymph node biopsy showed a high grade centroblastic B cell non-Hodgkin's lymphoma. She was classified as having bulky stage IIB disease and treated with combination chemotherapy, CHOP-methotrexate (cyclophospham-

Correspondence: M.E. Gore, Ph.D., F.R.C.P.

Accepted: 1 February 1994 ide $750 \mathrm{mg} / \mathrm{m}^{2}$ i.v. day 1 , doxorubicin $50 \mathrm{mg} / \mathrm{m}^{2}$ i.v. day 1 , vincristine $1.4 \mathrm{mg} / \mathrm{m}^{2}$ ( $2 \mathrm{mg}$ maximum) i.v. day 1 , methotrexate $250 \mathrm{mg} / \mathrm{m}^{2}$ on day 8 and prednisolone $100 \mathrm{mg}$ /day p.o. days $1-5$; cycle 21 days). Clinical and radiological complete remission was achieved after six courses and therefore two further cycles were given. Thus she received a cumulative dose of $400 \mathrm{mg} / \mathrm{m}^{2}$ of doxorubicin. Adjuvant radiotherapy was administered to the left axilla with fields well outside the cardiac contours to a total dose of $40 \mathrm{~Gy}$ given in 20 fractions.

Two months after completing chemotherapy the patient presented with a 3 week history of a flu-like illness, signs of biventricular failure and huge cardiomegaly. Electrocardiogram showed ventricular bigeminy with left axis deviation and echocardiography confirmed that both ventricles were enlarged with global poor contractility. A diagnosis of doxorubicin-induced cardiomyopathy was made and this was confirmed by endomyocardial biopsy. The patient was successfully treated with frusemide, nitrates and an angiotensin converting enzyme inhibitor; 5 months following the onset of cardiac failure the patient died of a cerebrovascular accident apparently disease-free from her lymphoma and without recurrence of her cardiac failure.

\section{Case 2}

A 14 year old girl of Ghanaian origin presented with a 2 month history of a $5 \mathrm{~cm}$ swelling in the right buccal mucosa. Biopsy of the lesion showed 
an embryonal rhabdomyosarcoma and staging investigations failed to reveal any metastases. She was treated with combination chemotherapy, VAC vincristine $1.4 \mathrm{mg} / \mathrm{m}^{2}$ ( $2 \mathrm{mg}$ maximum) i.v. day 1 , doxorubicin $50 \mathrm{mg} \mathrm{m}^{2}$ i.v. day 1, cyclophosphamide $600 \mathrm{mg} / \mathrm{m}^{2}$ i.v. day 1 ; cycle 7 days). Clinical complete remission was achieved after six courses and pathological complete remission was confirmed after the 8th and final course of treatment. She received a cumulative dose of $343 \mathrm{mg} / \mathrm{m}^{2}$ of doxorubicin, allowing for dose reductions due to changes in her body weight. Other than two episodes of neutropenic sepsis, treated with standard antibiotics, chemotherapy was well tolerated.

Three months after completion of treatment she presented with dyspnoea and worsening exercise tolerance. Clinically, she had severe biventricular failure and her chest X-ray showed features compatible with a dilated cardiomyopathy. Despite intensive treatment with nitrates, angiotensin converting enzyme inhibitors, loop diuretics and digoxin she continued to be symptomatic and underwent orthotopic cardiac transplantation. Two episodes of rejection following transplantation were successfully treated with pulsed methylprednisolone. The patient is alive and well with no evidence of tumour recurrent 7 years after transplantation, athough she remains on cyclosporine and azathioprine daily.

\section{Discussion}

The anthracycline group of drugs are widely used in the treatment of acute leukaemias, lymphomas, embryonal tumours and solid tumours such as breast and small cell lung cancer. Neither of our patients had any risk factors for myocardial disease but both developed significant cardiac failure after cumulative doxorubicin doses of 343 and $400 \mathrm{mg}$ / $\mathrm{m}^{2}$.

The incidence of symptomatic cardiac failure secondary to doxorubicin administration is dosedependent, although it was underestimated by early studies. ' Van Hoff et al. showed that the probability of developing congestive heart failure at a cumulative doxorubicin dose of $300 \mathrm{mg} / \mathrm{m}^{2}$ was between 0.7 and $3.4 \%$, depending on patient age, when the drug is administered on a conventional 3 weekly bolus regime. The probability rose to between $1.3 \%$ and $6.1 \%$, if a dose of $450 \mathrm{mg} / \mathrm{m}^{2}$ was given, and rose steeply at higher doses. ${ }^{2}$ The mortality rate from doxorubicin-induced cardiomyopathy is around $20-30 \%$ and symptoms of cardiac failure begin 1-2 months after the treatment though the interval can be as long as $2 \frac{1}{2}$ years. ${ }^{3,4}$ The development of congestive heart failure after low doses of doxorubicin is associated with a number of risk factors such as older age, family history of ischaemic heart disease, abnormal pre-chemotherapy electrocardiogram, prior exposure to other cardiotoxic agents and previous mediastinal irradiation. $2,4,5$

Invasive and noninvasive assessments of left ventricular function have been made in order to identify those at risk of developing cardiomyopathy after treatment with doxorubicin. Electrocardiography has been used as a screening method to detect early myocardial damage, but only nonspecific features are found and the changes often occur too late to be of clinical use. ${ }^{6}$ Radionuclide angiography shows a reduction in left ventricular ejection fraction after treatment with doxorubicin, the sensitivity being increased by exercising the subject. ${ }^{7.8}$ This technique can play a role in monitoring patients, although it is not universally available. The value of serial echocardiography to assess left ventricular function is unclear and remains to be fully evaluated; it was, however, used in both our patients to aid diagnosis. Subclinical impairment of myocardial function has been demonstrated in children treated with anthracylines, using post-exercise echocardiography, although the long-term significance of these findings is unclear. ${ }^{9}$

Electron microscopic examination of endomyocardial biopsies is considered by many to be necessary for a definitive diagnosis of doxorubicininduced myocardial damage. Bristow and colleagues ${ }^{5}$ found characteristic histological changes in 27 of 29 patients who had received more than $240 \mathrm{mg} / \mathrm{m}^{2}$ of doxorubicin although only seven patients had any clinical evidence of cardiomyopathy.

Three strategies have been suggested to overcome the problem of doxorubicin-induced cardiotoxicity. Firstly, the drug can be administered by slow intravenous infusion over periods of 48-96 hours. This results in significantly less clinical cardiotoxicity than conventional 3-weekly bolus injections and antimitotic activity is not impaired. ${ }^{10}$ Secondly, free-radical scavengers can offer partial protection against doxorubicin-induced cardiomyopathy if administered concomitantly with the drug." Finally, newer anthracycline analogues such as epirubicin have been developed with a similar spectrum of activity but which cause less cardiotoxicity than the parent compound. ${ }^{12}$

Both of our patients initially were managed by aggressive medical treatment but this failed in case 2 and she required cardiac transplantation. Cardiac transplantation for patients with a history of malignant disease has been successful, ${ }^{13}$ but it has rarely been performed for doxorubicin-induced cardiomyopathy and there are less than a dozen reported such cases. ${ }^{13-17}$

Our cases demonstrate that life-threatening cardiac failure may occur after conventional 'safe' 
doses of doxorubicin. In patients with potentially curable malignancies, doxorubicin-induced cardiomyopathy should be treated aggressively, as though the cardiomyopathy were their only prob- lem. These cases also highlight the current disquiet over the long-term cardiotoxic effects of doxorubicin as more children and young adults who have been exposed to this drug are cured.

\section{References}

1. Minow, R.A., Benjamin, R.S. \& Gottlieb, J.A. Adriamycin (NSC-123127), cardiomyopathy-an overview with determination of risk factors. Cancer Chemother Rep 1975, 6: 195-201.

2. Von Hoff, D.D., Layard, M.W., Basa, P. et al. Risk factors for doxorubicin-induced congestive heart failure. Ann Intern Med 1979, 91: 710-717.

3. Haq, M.M., Lagha, S.S., Choksi, J. et al. Doxorubicininduced congestive heart failure in adults. Cancer 1985, 56: $1361-1365$.

4. Buzdar, A.U., Marcus, C., Smith, T.L. \& Blumenschein, G.R. Early and delayed clinical cardiotoxicity of doxorubicin. Cancer 1985, 55: 2761-2765.

5. Bristow, M.R., Mason, J.W., Billingham, M.E. \& Daniels, J.R. Doxorubicin cardiomyopathy: evaluation by phonocardiography, endomyocardial biopsy, and cardiac catheterization. Ann Intern Med 1978, 88: 168-175.

6. Ali, M.K., Soto, A., Maroongroge, D. et al. Electrocardiographic changes after adriamycin chemotherapy. Cancer 1979, 43: 465-471.

7. Palmeri, S.T., Bonow, R.O., Myers, C.E. et al. Prospective evaluation of doxorubicin cardiotoxicity by rest and exercise radionuclide angiography. Am J Cardiol 1986, 58: 607-613.

8. McKillop, J.H., Bristow, M.R., Goris, M.L., Billingham, M.E. \& Bockenmuehl, K. Sensitivity and specificity of radionuclide ejection fractions in doxorubicin cardiotoxicity. Am Heart J 1983, 106: 1048-1056.

9. Yeung, S.T., Yoony, C., Spink, J., Galbraith, A.\& Smith, P.J. Functional myocardial impairment in children treated with anthracyclines for cancer. Lancet 1991, 337: 816-818.

10. Hortobagyi, G.N., Frye, D., Burzdar, A.U. et al. Decreased cardiotoxicity of doxorubicin administered by continuous intravenous infusion in combination chemotherapy for metastatic breast carcinoma. Cancer 1989, 63: 37-45.

11. Speyer, J.L., Green, M.D., Kramer, E. et al. Protective effect of the bispiperazinedione ICRF-187 against doxorubicin induced cardiac toxicity in women with advanced breast cancer. $N$ Engl J Med 1988, 319: 745-752.

12. Neri, B., Cini-Neri, G., Bandinelli, M. et al. Doxorubicin and epirubicin cardiotoxicity: experimental and clinical aspects. Int J Clin Pharmacol Ther Toxicol 1989, 27: 217-221.

13. Armitage, J.M., Kormos, R.L., Griffith, B.P., Fricker, F.J. \& Hardesty, R.L. Heart transplantation in patients with malignant disease. J Heart Transplant 1990, 9: 627-630.

14. Arico, M., Nespoli, L., Pedroni, E., Bonelti, F., Vigano, M. \& Burgio, G.R. Heart transplantation in a child with doxorubicin induced cardiomyopathy. $N$ Engl J Med 1988, 319: 1353.

15. Aldouri, M.A., Lopes, M.E.G., Yacoub, M. et al. Cardiac transplantation for doxorubicin-induced cardiomyopathy in acute myeloid leukaemia. Br J Haem 1990, 74: 541.

16. Luthy, A., Furrer, M., Waser, M. et al. Orthotopic heart transplantation: an efficient treatment in a young boy with doxorubicin-induced cardiomyopathy. $J$ Heart Lung Transplant 1992, 11: 815-816.

17. McManus, R.P. \& O'Hair, D.P. Pediatric heart transplantation for doxorubicin-induced cardiomyopathy. J Heart Lung Transplant 1992, 11: 375-376. 